\title{
4
}

\section{Second Order Shear Deformation Theory (SSDT) for Free Vibration Analysis on a Functionally Graded Quadrangle Plate}

\author{
A. Shahrjerdi ${ }^{1}$ and F. Mustapha ${ }^{2}$ \\ ${ }^{1}$ Department of Mechanical Engineering, Malayer University, Malayer, \\ ${ }^{2}$ Department of Aerospace Engineering, Universiti Putra Malaysia, \\ 43400 UPM, Serdang, Selangor \\ ${ }^{1}$ Iran \\ ${ }^{2}$ Malaysia
}

\section{Introduction}

Studies of vibration of plates have matured and are a well-established branch of research in structural dynamics. They have a vast range of applications in engineering and technology. But not much work can be found on vibration analysis of Functionally Graded Materials (FGMs) as compared to isotropic and composite plates and shells. FGMs are those in which the volume fraction of the two or more constituent materials is varied, as a power-law distribution, continuously as a function of position along certain dimension(s) of the structure $[1,2]$.

From the perspective of finite element method (FEM) studies of FGM, Praveen and Reddy [3], studied the static and dynamic responses of functionally graded (FG) ceramic-metal plate accounting for the transverse shear deformation, rotary inertia and moderately large rotations in the Von-Karman sense, in which the effect of an imposed temperature field on the response of the FG plate was discussed in detail. $\mathrm{Ng}$ et al. [4] dealt with the parametric resonance of FG rectangular plates under harmonic in-plane loading. Ferreira and Batra [5] provided a global collocation method for natural frequencies of FG plates by a meshless method with first order shear deformation theory (FSDT). Woo et al. [6] presented an analytical solution for the nonlinear free vibration behavior of FGM plates, where the fundamental equations were obtained using the Von-Karman theory for large transverse deflection, and the solution was based in terms of mixed Fourier series. Zhao et al. [7] studied the free vibration analysis of metal and ceramic FG plates using the element-free kpRitz method. The FSDT was employed to account for the transverse shear strain and rotary inertia, mesh-free kernel particle functions were used to approximate the two-dimensional displacement fields and the eigen-equation was obtained by applying the Ritz procedure to the energy functional of the system. Batra and Jin [8] used the FSDT coupled with the FEM to study the free vibrations of an FG anisotropic rectangular plate with various edge conditions. Also, Batra and Aimmanee [9] studied a higher order shear and normal deformable plate theory by FEM. Many studies conducted on FGMs are related to the analysis of free vibration by applying FSDT (see [10-12] and the references there in).

Other forms of shear deformation theory, such as the third order-shear deformation theory (TSDT) that accounts for the transverse effects, have been considered. Cheng and Batra [13] 
applied Reddy's third order plate theory to study buckling and steady state vibrations of a simply supported FG isotropic polygonal plate [14]. Vel and Batra [14] dealt with the threedimensional exact solution for free and forced vibrations of simply supported FGM rectangular plates using FDST and TSDT by employing the power series method. Nonlinear vibration and dynamic response of FGM plates in thermal environments were studied by Huang et al. [15] based on the higher-order shear deformation plate theory and general VonKarman type equation. Static analysis of FG plates using TSDT and a meshless method were also presented by Ferreira et al. [16].

As for the first-order shear deformation plate theory (FSDT), the theory extends the kinematics of the classical plate theory $(\mathrm{CPT})$ by relaxing the normality restriction and allowing for arbitrary but constant rotation of transverse normals. On the other hand, the second and third order shear deformation plate theory further relaxes the kinematic hypothesis by removing the straightness assumption; i.e., the straight normal to the middle plane before deformation may become cubic curves after deformation. The most significant difference between the classical and shear deformation theories is the effect of including transverse shear deformation on the predicted deflections, frequencies, and buckling loads [19].

A unified derivation of various shear-deformation models consists of Kirchhoff-Love type, Mindlin-Reissner type theory, third order theory, Layer-Wise theory and Exact-Solution. Librescu et al. [22] studied the correlation between two apparently different higher-order theories and First order transverse shear deformation theory (FSDT) of anisotropic plates. The Kirchhoff-Love assumptions were developed by Librescu and Schmidt [23]. The theory incorporates normal and shear deformation (transverse) as well as the higher-order effects, and accounts for small strains and moderate rotations of the normal.

For experimental work, shear deformation validation and compared structural theories, Stoffle [20] measured and simulated vibrations of viscoplastic plates under impulsive loading and determined how accurately the measured deformations can be calculated by the chosen constitutive and structural theories. He assumed a first-order shear deformation shell theory and applied small strains and moderate rotations and viscoplastic laws. He applied short time measurement techniques to shock tubes in order to record fast loading processes and plate deformations.

As mentioned above, shear deformation theories have been applied to consider transverse shear strains and rotation. Axisymmetric bending and stretching of functionally graded solid circular and annular plates were studied using the second-order shear deformation plate theory by Saidi and Sahraee [21]. Khdeir and Reddy [17] studied the free vibration of laminated composite plates using SSDT. Bahtuei and Eslami [18] also investigated the coupled thermoelastic response of a FG circular cylindrical shell by considering SSDT.

To the authors' knowledge, not much work has been done in the area of the dynamic stability of FG plate by using SSDT. In this study, the free vibration of FG plates (rectangular and square) by using SSDT is presented. The material properties of the plates are graded along the thickness direction according to a volume fraction power law distribution. Classical elasticity is considered and the complete governing equations are presented. Navier's method is applied to solve the equations. This work aims to investigate the effect of some basic factors such as material properties, side-to-side and side-to-thickness ratio for FG quadrangular plates on simply supported boundary conditions.

\section{Gradation relations}

The most commonly used models for most of the literature that express the variation of material properties in FGMs is the power law distribution of the volume fraction. According 
to this model, the material property gradation through the thickness of the plate is assumed to be in the following form [10]:

$$
\begin{aligned}
& E=E\left(x_{3}\right)=\left(E_{c}-E_{m}\right)\left(x_{3} / h+1 / 2\right)^{p}+E_{m} \\
& \rho=\rho\left(x_{3}\right)=\left(\rho_{c}-\rho_{m}\right)\left(x_{3} / h+1 / 2\right)^{p}+\rho_{m}
\end{aligned}
$$

Here $E$ and $\rho$ denote the modulus of elasticity and density of FG structure, while the parameters with subscript $m$ or $c$ represent the material properties of a pure metal and pure ceramic plate, respectively. The thickness coordinate variable is presented by $x_{3}$ while $-\frac{h}{2} \leq x_{3} \leq \frac{h}{2}$, where $h$ is the total thickness of the plate as shown in Figure 1. $p \geq 0$ is the volume fraction exponent (also called grading index in this paper); $\left(x_{3} / h+1 / 2\right)^{p}$ denotes the volume fraction of the ceramic.
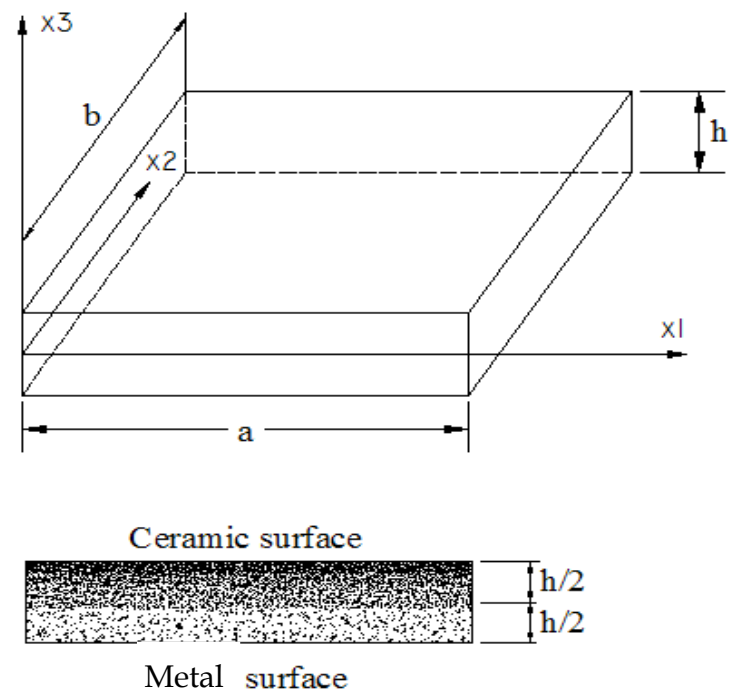

Fig. 1. Functionally graded plate.

A FG rectangular is considered as shown in Figure 1. The material in the top surface and in the bottom surface is Full-Ceramic and Full-Metal respectively, and between these two pure materials, the power law distribution of material is applied. The most well-known FGM is compositionally graded from a ceramic to a metal to incorporate such diverse properties as heat, wear and oxidation resistance of ceramics with the toughness, strength, machinability and bending capability of metals [7].

\section{Elastic equations}

Under consideration is a thin FG plate with constant thickness $h$, width, $a$, and length , $b$, as shown in Figure 1. Cartesian coordinate system $\left(x_{1}, x_{2}, x_{3}\right)$ is used. 


\subsection{Displacement field and strains}

The SSDT is based on the following representation of the displacement field:

$$
\begin{gathered}
u_{1}=u+x_{3} \phi_{1}+x_{3}^{2} \phi_{2} \\
u_{2}=v+x_{3} \psi_{1}+x_{3}^{2} \psi_{2} \\
u_{3}=w
\end{gathered}
$$

Where $\left(u_{1}, u_{2}, u_{3}\right)$ denote the displacement components in the $\left(x_{1}, x_{2}, x_{3}\right)$ directions respectively; $(u, v, w)$ are the displacements of a point on the mid plane $\left(x_{1}, x_{2}, 0\right)$. All displacement components $\left(u, v, w, \phi_{1}, \phi_{2}, \psi_{1}, \psi_{2}\right)$ are functions of position $\left(x_{1}, x_{2}\right)$ and time $t$. The strain-displacement equations of the linear strain are given by [19].

$$
\begin{gathered}
\left\{\begin{array}{l}
\varepsilon_{11} \\
\varepsilon_{22} \\
\gamma_{12}
\end{array}\right\}=\left\{\begin{array}{l}
\varepsilon_{11}^{0} \\
\varepsilon_{22}^{0} \\
\varepsilon_{12}^{0}
\end{array}\right\}+x_{3}\left\{\begin{array}{l}
\kappa_{11} \\
\kappa_{22} \\
\kappa_{12}
\end{array}\right\}+x_{3}^{2}\left\{\begin{array}{c}
\kappa_{11}^{\prime} \\
\kappa_{22}^{\prime} \\
\kappa_{12}^{\prime}
\end{array}\right\} \\
\left\{\begin{array}{l}
\gamma_{23} \\
\gamma_{13}
\end{array}\right\}=\left\{\begin{array}{l}
\gamma_{23}^{0} \\
\gamma_{13}^{0}
\end{array}\right\}+x_{3}\left\{\begin{array}{l}
\gamma_{23}^{1} \\
\gamma_{13}^{1}
\end{array}\right\}
\end{gathered}
$$

where

$$
\begin{aligned}
& \varepsilon_{11}^{0}=\frac{\partial u}{\partial x_{1}}, \kappa_{11}=\frac{\partial \phi_{1}}{\partial x_{1}}, \kappa_{11}^{\prime}=\frac{\partial \phi_{2}}{\partial x_{1}} \\
& \varepsilon_{22}^{0}=\frac{\partial v}{\partial x_{2}}, \kappa_{22}=\frac{\partial \psi_{1}}{\partial x_{2}}, \kappa_{22}^{\prime}=\frac{\partial \psi_{2}}{\partial x_{2}} \\
& \varepsilon_{12}^{0}=\frac{\partial u}{\partial x_{2}}+\frac{\partial v}{\partial x_{1}}, \kappa_{12}=\frac{\partial \phi_{1}}{\partial x_{2}}+\frac{\partial \psi_{1}}{\partial x_{1}}, \kappa_{12}^{\prime}=\frac{\partial \phi_{2}}{\partial x_{2}}+\frac{\partial \psi_{2}}{\partial x_{1}} \\
& \gamma_{23}^{0}=\psi_{1}+\frac{\partial w}{\partial x_{2}}, \gamma_{13}^{0}=\phi_{1}+\frac{\partial w}{\partial x_{1}}, \gamma_{23}^{1}=2 \psi_{2}, \gamma_{13}^{1}=2 \phi_{2}
\end{aligned}
$$

\subsection{Stress-strain relations}

The stress-strain relations are given by $[17,19]$.

$$
\left\{\begin{array}{l}
\sigma_{11} \\
\sigma_{22} \\
\sigma_{23} \\
\sigma_{13} \\
\sigma_{12}
\end{array}\right\}=\left\{\begin{array}{ccccc}
q_{11} & q_{12} & 0 & 0 & 0 \\
q_{12} & q_{22} & 0 & 0 & 0 \\
0 & 0 & q_{44} & 0 & 0 \\
0 & 0 & 0 & q_{55} & 0 \\
0 & 0 & 0 & 0 & q_{66}
\end{array}\right\}\left\{\begin{array}{l}
\varepsilon_{11} \\
\varepsilon_{22} \\
\gamma_{23} \\
\gamma_{13} \\
\gamma_{12}
\end{array}\right\}
$$

where $q_{i j}$ are the material constants given by 


$$
q_{11}=q_{22}=\frac{E}{1-v^{2}} \quad q_{12}=v q_{11} \quad q_{44}=q_{55}=q_{66}=\frac{E}{2(1+v)}
$$

Hence, it follows that

$$
\left\{\begin{array}{l}
\sigma_{11} \\
\sigma_{22} \\
\sigma_{23} \\
\sigma_{13} \\
\sigma_{12}
\end{array}\right\}=\left[\begin{array}{ccccc}
q_{11} & q_{12} & 0 & 0 & 0 \\
q_{12} & q_{22} & 0 & 0 & 0 \\
0 & 0 & q_{66} & 0 & 0 \\
0 & 0 & 0 & q_{55} & 0 \\
0 & 0 & 0 & 0 & q_{44}
\end{array}\right]\left(\left\{\begin{array}{l}
\varepsilon_{11}^{0} \\
\varepsilon_{22}^{0} \\
\gamma_{23}^{0} \\
\gamma_{13}^{0} \\
\varepsilon_{12}^{0}
\end{array}\right\}+x_{3}\left\{\begin{array}{l}
\kappa_{11} \\
\kappa_{22} \\
\gamma_{23}^{1} \\
\gamma_{13}^{1} \\
\kappa_{12}
\end{array}\right\}+x_{3}^{2}\left\{\begin{array}{l}
\kappa_{11}^{\prime} \\
\kappa_{22}^{\prime} \\
0 \\
0 \\
\kappa_{12}^{\prime}
\end{array}\right\}\right)
$$

\subsection{Equations of motion}

For the case of a rectangular plate, $K, U$ and $V$ are the kinetic, strain and potential energies of the body, respectively. The summation of the potential energy of external forces and strain energy, $U+V$, is the total potential energy, $\Pi$, of the body. Hamilton's principle for an elastic body is given by,

$$
\int_{t_{1}}^{t_{2}}(\delta K-\delta \Pi) d t=0
$$

The inertias are defined by

$$
I_{i}=\int_{\frac{-h}{2}}^{\frac{h}{2}} \rho_{0}\left(x_{3}\right)^{i} d x_{3} \quad(i=0,1,2, \ldots, 6)
$$

Hamilton's principle, equation (8), along with the SSDT, given by equation (2), yields the complete form of the equilibrium equations:

$$
\begin{aligned}
& \frac{\partial Q_{13}}{\partial x_{1}}+\frac{\partial Q_{23}}{\partial x_{2}}=I_{0} \ddot{w} \\
& \frac{\partial M_{11}}{\partial x_{1}}+\frac{\partial M_{12}}{\partial x_{2}}-Q_{13}=I_{2} \ddot{\phi}_{1}+I_{1} \ddot{u}+I_{3} \ddot{\phi}_{2} \\
& \frac{\partial L_{11}}{\partial x_{1}}+\frac{\partial L_{12}}{\partial x_{2}}-2 R_{13}=I_{2} \ddot{u}+I_{4} \ddot{\phi}_{2}+I_{3} \ddot{\phi}_{1} \\
& \frac{\partial M_{22}}{\partial x_{2}}+\frac{\partial M_{12}}{\partial x_{1}}-Q_{23}=I_{2} \ddot{\psi}_{1}+I_{1} \ddot{v}+I_{3} \ddot{\psi}_{2} \\
& \frac{\partial L_{22}}{\partial x_{2}}+\frac{\partial L_{12}}{\partial x_{1}}-2 R_{23}=I_{2} \ddot{v}+I_{4} \ddot{\psi}_{2}+I_{3} \ddot{\psi}_{1}
\end{aligned}
$$

where $N, M, L, Q$ and $R$ are the stress resultants. These parameters can be represented by 


$$
\begin{aligned}
& \left\{\begin{array}{l}
N_{11} \\
N_{22} \\
N_{12}
\end{array}\right\}=\int_{\frac{-h}{2}}^{\frac{h}{2}}\left\{\begin{array}{l}
\sigma_{11} \\
\sigma_{22} \\
\sigma_{12}
\end{array}\right\} d x_{3}\left\{\begin{array}{l}
N_{11} \\
N_{22} \\
N_{12}
\end{array}\right\}=\left[\begin{array}{c}
A_{11} \varepsilon_{11}^{0}+B_{11} \kappa_{11}+D_{11} \kappa_{11}^{\prime}+A_{12} \varepsilon_{22}^{0}+B_{12} \kappa_{22}+D_{12} \kappa_{22}^{\prime} \\
A_{12} \varepsilon_{11}^{0}+B_{12} \kappa_{11}+D_{12} \kappa_{11}^{\prime}+A_{22} \varepsilon_{22}^{0}+B_{22} \kappa_{22}+D_{22} \kappa_{22}^{\prime} \\
A_{66} \varepsilon_{12}^{0}+B_{66} \kappa_{12}+D_{66} \kappa_{12}^{\prime}
\end{array}\right] \\
& \left\{\begin{array}{l}
M_{11} \\
M_{22} \\
M_{12}
\end{array}\right\}=\int_{\frac{-h}{2}}^{\frac{h}{2}}\left\{\begin{array}{l}
\sigma_{11} \\
\sigma_{22} \\
\sigma_{12}
\end{array}\right\} x_{3} d x_{3}\left\{\begin{array}{l}
M_{11} \\
M_{22} \\
M_{12}
\end{array}\right\}=\left[\begin{array}{c}
B_{11} \varepsilon_{11}^{0}+D_{11} \kappa_{11}+E_{11} \kappa_{11}^{\prime}+B_{12} \varepsilon_{22}^{0}+D_{12} \kappa_{22}+E_{12} \kappa_{22}^{\prime} \\
B_{12} \varepsilon_{11}^{0}+D_{12} \kappa_{11}+E_{12} \kappa_{11}^{\prime}+B_{22} \varepsilon_{22}^{0}+D_{22} \kappa_{22}+E_{22} \kappa_{22}^{\prime} \\
B_{66} \varepsilon_{12}^{0}+D_{66} \kappa_{12}+E_{66} \kappa_{12}^{\prime}
\end{array}\right] \\
& \left\{\begin{array}{l}
L_{11} \\
L_{22} \\
L_{12}
\end{array}\right\}=\int_{\frac{-h}{2}}^{\frac{h}{2}}\left\{\begin{array}{l}
\sigma_{11} \\
\sigma_{22} \\
\sigma_{12}
\end{array}\right\} x^{2}{ }_{3} d x_{3}\left\{\begin{array}{l}
L_{11} \\
L_{22} \\
L_{12}
\end{array}\right\}=\left[\begin{array}{c}
D_{11} \varepsilon_{11}^{0}+E_{11} \kappa_{11}+F_{11} \kappa_{11}^{\prime}+D_{12} \varepsilon_{22}^{0}+E_{12} \kappa_{22}+F_{12} \kappa_{22}^{\prime} \\
D_{12} \varepsilon_{11}^{0}+E_{12} \kappa_{11}+F_{12} \kappa_{11}^{\prime}+D_{22} \varepsilon_{22}^{0}+E_{22} \kappa_{22}+F_{22} \kappa_{22}^{\prime} \\
D_{66} \varepsilon_{12}^{0}+E_{66} \kappa_{12}+F_{66} \kappa_{12}^{\prime}
\end{array}\right] \\
& \left\{\begin{array}{l}
Q_{13} \\
Q_{23}
\end{array}\right\}=\int_{\frac{-h}{2}}^{\frac{h}{2}}\left\{\begin{array}{l}
\sigma_{13} \\
\sigma_{23}
\end{array}\right\} d x_{3},\left\{\begin{array}{l}
Q_{13} \\
Q_{23}
\end{array}\right\}=\left[\begin{array}{l}
A_{55} \gamma_{13}^{0}+B_{55} \gamma_{13}^{1} \\
A_{44} \gamma_{23}^{0}+B_{44} \gamma_{23}^{1}
\end{array}\right] \\
& \left\{\begin{array}{l}
R_{13} \\
R_{23}
\end{array}\right\}=\int_{\frac{-h}{2}}^{\frac{h}{2}}\left\{\begin{array}{l}
\sigma_{13} \\
\sigma_{23}
\end{array}\right\} x_{3} d x_{3},\left\{\begin{array}{l}
R_{13} \\
R_{23}
\end{array}\right\}=\left[\begin{array}{l}
B_{55} \gamma_{13}^{0}+D_{55} \gamma_{13}^{1} \\
B_{44} \gamma_{23}^{0}+D_{44} \gamma_{23}^{1}
\end{array}\right]
\end{aligned}
$$

where

$$
A_{i j}, B_{i j}, D_{i j}, E_{i j}, F_{i j}=\int_{\frac{-h}{2}}^{\frac{h}{2}} q_{i j}\left(1, x_{3}, x_{3}^{2}, x_{3}^{3}, x_{3}^{4}\right) d x_{3}
$$

Here $A_{i j}, B_{i j}, D_{i j}, E_{i j}$ and $F_{i j}$ are the plate stiffnesses.

$$
\text { For }\left\{\begin{array}{lr}
A_{i j}, D_{i j}, F_{i j} & (i, j=1,2,4,5,6) \\
E_{i j}, B_{i j} & (i, j=1,2,6)
\end{array}\right.
$$

By substituting equation (4) into equation (11) and then into equation (10) and also by applying definition (12), Navier's equations for FG plates are obtained as follows:

$$
\begin{aligned}
& A_{11} \frac{\partial^{2} u}{\partial x_{1}^{2}}+A_{66} \frac{\partial^{2} u}{\partial x_{2}^{2}}+\left(A_{12}+A_{66}\right) \frac{\partial^{2} v}{\partial x_{1} \partial x_{2}}+B_{11} \frac{\partial^{2} \phi_{1}}{\partial x_{1}^{2}}+B_{66} \frac{\partial^{2} \phi_{1}}{\partial x_{2}^{2}}+D_{11} \frac{\partial^{2} \phi_{2}}{\partial x_{1}^{2}}+ \\
& D_{66} \frac{\partial^{2} \phi_{2}}{\partial x_{2}^{2}}+\left(B_{12}+B_{66}\right) \frac{\partial^{2} \psi_{1}}{\partial x_{1} \partial x_{2}}+\left(D_{12}+D_{66}\right) \frac{\partial^{2} \psi_{2}}{\partial x_{1} \partial x_{2}}=I_{0} \ddot{u}+I_{2} \ddot{\phi}_{2}+I_{1} \ddot{\phi}_{1}
\end{aligned}
$$




$$
\begin{aligned}
& A_{12} \frac{\partial^{2} u}{\partial x_{1} \partial x_{2}}+A_{66} \frac{\partial^{2} u}{\partial x_{1} \partial x_{2}}+A_{22} \frac{\partial^{2} v}{\partial x_{2}^{2}}+A_{66} \frac{\partial^{2} v}{\partial x_{1}^{2}}+\left(B_{12}+B_{66}\right) \frac{\partial^{2} \phi_{1}}{\partial x_{1} \partial x_{2}}+\left(D_{12}+D_{66}\right) \frac{\partial^{2} \phi_{2}}{\partial x_{1} \partial x_{2}} \\
& +B_{66} \frac{\partial^{2} \psi_{1}}{\partial x_{1}^{2}}+B_{22} \frac{\partial^{2} \psi_{1}}{\partial x_{2}^{2}}+D_{66} \frac{\partial^{2} \psi_{2}}{\partial x_{1}^{2}}+D_{22} \frac{\partial^{2} \psi_{2}}{\partial x_{2}^{2}}=I_{0} \ddot{v}+I_{2} \ddot{\psi}_{2}+I_{1} \ddot{\psi}_{1} \\
& A_{55} \frac{\partial \phi_{1}}{\partial x_{1}}+A_{55} \frac{\partial^{2} w}{\partial x_{1}{ }^{2}}+2 B_{55} \frac{\partial \phi_{2}}{\partial x_{1}}+A_{44} \frac{\partial \psi_{1}}{\partial x_{2}}+A_{44} \frac{\partial^{2} w}{\partial x_{2}{ }^{2}}+2 B_{44} \frac{\partial \psi_{2}}{\partial x_{2}}=I_{0} \ddot{w} \\
& B_{11} \frac{\partial^{2} u}{\partial x_{1}^{2}}+B_{66} \frac{\partial^{2} u}{\partial x_{2}^{2}}+\left(B_{12}+B_{66}\right) \frac{\partial^{2} v}{\partial x_{1} \partial x_{2}}+D_{11} \frac{\partial^{2} \phi_{1}}{\partial x_{1}^{2}}+D_{66} \frac{\partial^{2} \phi_{1}}{\partial x_{2}^{2}} \\
& +E_{11} \frac{\partial^{2} \phi_{2}}{\partial x_{1}^{2}}+E_{66} \frac{\partial^{2} \phi_{2}}{\partial x_{2}^{2}}+\left(D_{12}+D_{66}\right) \frac{\partial^{2} \psi_{1}}{\partial x_{1} \partial x_{2}}+\left(E_{12}+E_{66}\right) \frac{\partial^{2} \psi_{2}}{\partial x_{1} \partial x_{2}} \\
& -A_{55}\left(\frac{\partial w}{\partial x_{1}}\right)-A_{55} \phi_{1}-2 B_{55} \phi_{2}=I_{2} \ddot{\phi}_{1}+I_{1} \ddot{u}+I_{3} \ddot{\phi}_{2} \\
& D_{11} \frac{\partial^{2} u}{\partial x_{1}^{2}}+D_{66} \frac{\partial^{2} u}{\partial x_{2}^{2}}+\left(D_{12}+D_{66}\right) \frac{\partial^{2} v}{\partial x_{1} \partial x_{2}}+E_{11} \frac{\partial^{2} \phi_{1}}{\partial x_{1}^{2}}+E_{66} \frac{\partial^{2} \phi_{1}}{\partial x_{2}^{2}}+F_{11} \frac{\partial^{2} \phi_{2}}{\partial x_{1}^{2}}+F_{66} \frac{\partial^{2} \phi_{2}}{\partial x_{2}^{2}} \\
& +\left(E_{12}+E_{66}\right) \frac{\partial^{2} \psi_{1}}{\partial x_{1} \partial x_{2}}+\left(F_{12}+F_{66}\right) \frac{\partial^{2} \psi_{2}}{\partial x_{1} \partial x_{2}}-2\left(B_{55}\left(\frac{\partial w}{\partial x_{1}}\right)+2 D_{55} \phi_{2}+B_{55} \phi_{1}\right) \\
& =I_{2} \ddot{u}+I_{4} \ddot{\phi}_{2}+I_{3} \ddot{\phi}_{1} \\
& \left(B_{12}+B_{66}\right) \frac{\partial^{2} u}{\partial x_{1} \partial x_{2}}+B_{22} \frac{\partial^{2} v}{\partial x_{2}^{2}}+B_{66} \frac{\partial^{2} v}{\partial x_{1}^{2}}-A_{44} \frac{\partial w}{\partial x_{2}}+\left(D_{12}+D_{66}\right) \frac{\partial^{2} \phi_{1}}{\partial x_{1} \partial x_{2}}+ \\
& \left(E_{12}+E_{66}\right) \frac{\partial^{2} \phi_{2}}{\partial x_{1} \partial x_{2}}+D_{66} \frac{\partial^{2} \psi_{1}}{\partial x_{1}^{2}}+D_{22} \frac{\partial^{2} \psi_{1}}{\partial x_{2}^{2}}+E_{66} \frac{\partial^{2} \psi_{2}}{\partial x_{1}^{2}}+E_{22} \frac{\partial^{2} \psi_{2}}{\partial x_{2}^{2}} \\
& -A_{44} \psi_{1}-2 B_{44} \psi_{2}=I_{2} \ddot{\psi}_{1}+I_{1} \ddot{v}+I_{3} \ddot{\psi}_{2} \\
& \left(D_{12}+D_{66}\right) \frac{\partial^{2} u}{\partial x_{1} \partial x_{2}}+D_{66} \frac{\partial^{2} v}{\partial x_{1}^{2}}+D_{22} \frac{\partial^{2} v}{\partial x_{2}^{2}}-2 B_{44} \frac{\partial w}{\partial x_{2}}+\left(E_{12}+E_{66}\right) \frac{\partial^{2} \phi_{1}}{\partial x_{1} \partial x_{2}} \\
& +\left(F_{12}+F_{66}\right) \frac{\partial^{2} \phi_{2}}{\partial x_{1} \partial x_{2}}+E_{66} \frac{\partial^{2} \psi_{1}}{\partial x_{1}^{2}}+E_{22} \frac{\partial^{2} \psi_{1}}{\partial x_{2}^{2}}+F_{66} \frac{\partial^{2} \psi_{2}}{\partial x_{1}^{2}}+F_{22} \frac{\partial^{2} \psi_{2}}{\partial x_{2}^{2}} \\
& -2 B_{44} \psi_{1}-4 D_{44} \psi_{2}=I_{2} \ddot{v}+I_{4} \ddot{\psi}_{2}+I_{3} \ddot{\psi}_{1}
\end{aligned}
$$

It can be noted by considering zero values for $\phi_{2} \& \psi_{2}$ in equations (10) and (13), the FSDT equations can be obtained [19].

\section{Boundary conditions}

For the case of simply supported boundary conditions of FG, as shown in Figure 2, the following relations can be written: 


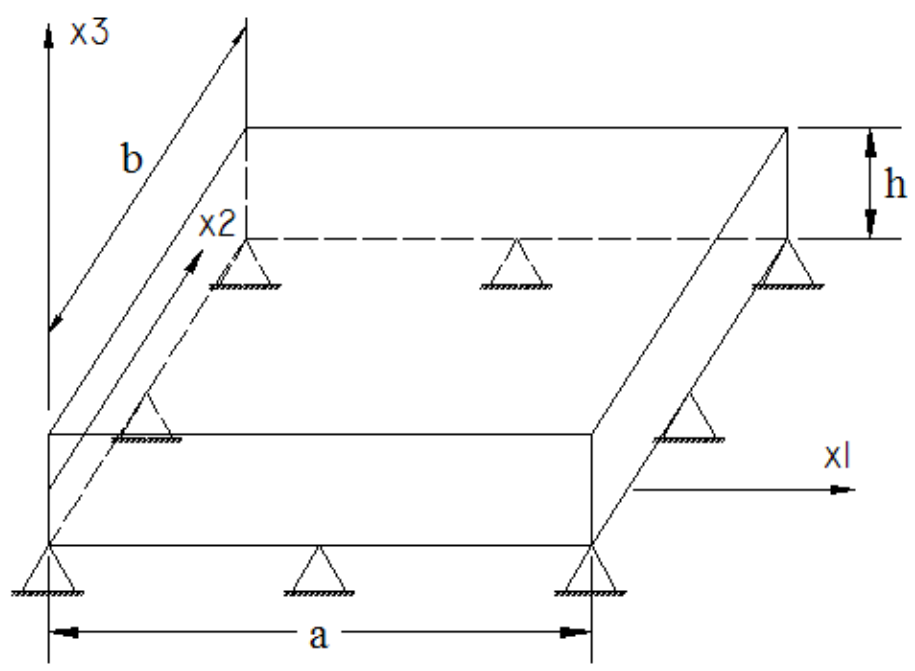

Fig. 2. Simply supported boundary condition in FG plates.

$$
\begin{aligned}
& x_{1}=0, a \Rightarrow\left\{\begin{array}{l}
v\left(0, x_{2}, t\right)=0 \\
v\left(a, x_{2}, t\right)=0 \\
\psi_{1}\left(0, x_{2}, t\right)=0 \\
\psi_{1}\left(a, x_{2}, t\right)=0 \\
\psi_{2}\left(0, x_{2}, t\right)=0 \\
\psi_{2}\left(a, x_{2}, t\right)=0
\end{array},\left\{\begin{array}{l}
M_{11}\left(0, x_{2}, t\right)=0 \\
N_{11}\left(0, x_{2}, t\right)=0 \\
M_{11}\left(a, x_{2}, t\right)=0 \\
N_{11}\left(a, x_{2}, t\right)=0
\end{array},\left\{\begin{array}{l}
w\left(0, x_{2}, t\right)=0 \\
w\left(a, x_{2}, t\right)=0
\end{array}\right.\right.\right. \\
& x_{2}=0, b \Rightarrow\left\{\begin{array}{l}
u\left(x_{1}, 0, t\right)=0 \\
u\left(x_{1}, b, t\right)=0 \\
\phi_{1}\left(x_{1}, 0, t\right)=0 \\
\phi_{1}\left(x_{1}, b, t\right)=0 \\
\phi_{2}\left(x_{1}, 0, t\right)=0 \\
\phi_{2}\left(x_{1}, b, t\right)=0
\end{array}, \begin{array}{l}
M_{22}\left(x_{1}, 0, t\right)=0 \\
N_{22}\left(x_{1}, 0, t\right)=0 \\
M_{22}\left(x_{1}, b, t\right)=0 \\
N_{22}\left(x_{1}, b, t\right)=0
\end{array},\left\{\begin{array}{l}
w\left(x_{1}, 0, t\right)=0 \\
w\left(x_{1}, b, t\right)=0
\end{array}\right.\right.
\end{aligned}
$$

\section{Method of solution}

The Navier method is used for frequency analysis of a simply supported FG plate. The displacement field can be assumed to be given by:

$$
u=\sum_{n=1}^{\infty} \sum_{m=1}^{\infty} u_{m n}(t) \cos \alpha x_{1} \sin \beta x_{2} \quad, u_{m n}(t)=U e^{-i \omega t}
$$




$$
\begin{aligned}
& v=\sum_{n=1}^{\infty} \sum_{m=1}^{\infty} v_{m n}(t) \sin \alpha x_{1} \cos \beta x_{2} \quad, v_{m n}(t)=V e^{-i \omega t} \\
& w=\sum_{n=1}^{\infty} \sum_{m=1}^{\infty} w_{m n}(t) \sin \alpha x_{1} \sin \beta x_{2} \quad, w_{m n}(t)=W e^{-i \omega t} \\
& \phi_{1}=\sum_{n=1}^{\infty} \sum_{m=1}^{\infty} \phi_{1 m n}(t) \cos \alpha x_{1} \sin \beta x_{2} \quad, \phi_{1 m n}(t)=\Phi_{1} e^{-i \omega t} \\
& \phi_{2}=\sum_{n=1}^{\infty} \sum_{m=1}^{\infty} \phi_{2 m n}(t) \cos \alpha x_{1} \sin \beta x_{2} \quad, \phi_{2 m n}(t)=\Phi_{2} e^{-i \omega t} \\
& \psi_{1}=\sum_{n=1}^{\infty} \sum_{m=1}^{\infty} \psi_{1 m n}(t) \sin \alpha x_{1} \cos \beta x_{2} \quad, \psi_{1 m n}(t)=\Psi_{1} e^{-i \omega t} \\
& \psi_{2}=\sum_{n=1}^{\infty} \sum_{m=1}^{\infty} \psi_{2 m n}(t) \sin \alpha x_{1} \cos \beta x_{2} \quad, \psi_{2 m n}(t)=\Psi_{2} e^{-i \omega t}
\end{aligned}
$$

where

$$
\alpha=\frac{m \pi}{a} \quad, \beta=\frac{n \pi}{b}
$$

For natural vibrations, substituting equation (15) into the equations of motion (13), these equations reduce to the following forms:

$$
[C]\left\{\begin{array}{l}
U \\
V \\
W \\
\Phi_{1} \\
\Phi_{2} \\
\psi_{1} \\
\psi_{2}
\end{array}\right\}-\omega^{2}[M]\left\{\begin{array}{c}
U \\
V \\
W \\
\Phi_{1} \\
\Phi_{2} \\
\psi_{1} \\
\psi_{2}
\end{array}\right\}=\left\{\begin{array}{l}
0 \\
0 \\
0 \\
0 \\
0 \\
0 \\
0
\end{array}\right\}
$$

where $\omega$ is the natural frequency and

$$
\begin{array}{lll}
C_{11}=A_{11} \alpha^{2}+A_{66} \beta^{2} & C_{21}=\left(A_{12}+A_{66}\right) \alpha \beta & C_{31}=0 \\
C_{12}=\left(A_{12}+A_{66}\right) \alpha \beta & C_{22}=A_{66} \alpha^{2}+A_{22} \beta^{2} & C_{32}=0 \\
C_{13}=0 & C_{23}=0 & C_{33}=A_{55} \alpha^{2}+A_{44} \beta^{2} \\
C_{14}=B_{11} \alpha^{2}+B_{66} \beta^{2} & C_{24}=\left(B_{12}+B_{66}\right) \alpha \beta & C_{34}=A_{55} \alpha \\
C_{15}=D_{11} \alpha^{2}+D_{66} \beta^{2} & C_{25}=\left(D_{12}+D_{66}\right) \alpha \beta & C_{35}=2 B_{55} \alpha \\
C_{16}=\left(B_{12}+B_{66}\right) \alpha \beta & C_{26}=B_{66} \alpha^{2}+B_{22} \beta^{2} & C_{36}=A_{44} \beta \\
C_{17}=\left(D_{12}+D_{66}\right) \alpha \beta & C_{27}=D_{66} \alpha^{2}+D_{22} \beta^{2} & C_{37}=2 B_{44} \beta
\end{array}
$$




$$
\begin{array}{ll}
C_{41}=B_{11} \alpha^{2}+B_{66} \beta^{2} & C_{51}=D_{11} \alpha^{2}+D_{66} \beta^{2} \\
C_{42}=\left(B_{12}+B_{66}\right) \alpha \beta & C_{52}=\left(D_{12}+D_{66}\right) \alpha \beta \\
C_{43}=A_{55} \alpha & C_{53}=2 B_{55} \alpha \\
C_{44}=D_{11} \alpha^{2}+D_{66} \beta^{2}+A_{55}, C_{54}=E_{11} \alpha^{2}+E_{66} \beta^{2}+2 B_{55} \\
C_{45}=E_{11} \alpha^{2}+E_{66} \beta^{2}+2 B_{55} & C_{55}=F_{11} \alpha^{2}+F_{66} \beta^{2}+4 D_{55} \\
C_{46}=\left(D_{12}+D_{66}\right) \alpha \beta & C_{56}=\left(E_{12}+E_{66}\right) \alpha \beta \\
C_{47}=\left(E_{12}+E_{66}\right) \alpha \beta & C_{57}=\left(F_{12}+F_{66}\right) \alpha \beta \\
C_{61}=\left(B_{12}+B_{66}\right) \alpha \beta & C_{71}=\left(D_{12}+D_{66}\right) \alpha \beta \\
C_{62}=B_{66} \alpha^{2}+B_{22} \beta^{2} & C_{72}=D_{66} \alpha^{2}+D_{22} \beta^{2} \\
C_{63}=A_{44} \beta & C_{73}=2 B_{44} \beta \\
C_{64}=\left(D_{12}+D_{66}\right) \alpha \beta & , C_{74}=\left(E_{12}+E_{66}\right) \alpha \beta \\
C_{65}=\left(E_{12}+E_{66}\right) \alpha \beta & C_{75}=\left(F_{12}+F_{66}\right) \alpha \beta \\
C_{66}=D_{66} \alpha^{2}+D_{22} \beta^{2}+A_{44} & C_{76}=E_{66} \alpha^{2}+E_{22} \beta^{2}+2 B_{44} \\
C_{67}=E_{66} \alpha^{2}+E_{22} \beta^{2}+2 B_{44} & C_{77}=F_{66} \alpha^{2}+F_{22} \beta^{2}+4 D_{44}
\end{array}
$$

By considering relations (18), equation (17) can be written as:

$$
\left|C_{i j}-M_{i j} \omega^{2}\right|=0
$$

By solving equation (19) and considering appropriate values for $\mathrm{n}$ and $\mathrm{m}$ in equation (16) the fundamental frequency of a quadrangle FG plate can be obtained.

\section{Validation and numerical results}

\subsection{Validation}

The results obtained for a FG plate by applying SSDT are compared with the results obtained by using TSDT as in Ref [5] and the exact solution of [14]. The following nondimensional fundamental frequencies in Table 1 and Table 2 are obtained by considering material properties the same as [5].

Results in Table 1 and Table 2 show that the values obtained by SSDT are greater than those from TSDT and the exact solution. This is due to the fact that the transverse shear and rotary inertia will have more of an effect on a thicker plate. For the thick plates considered in this

\begin{tabular}{|c|c|c|c|c|c|c|c|c|}
\hline \multicolumn{3}{|c|}{$h / a=0.05$} & \multicolumn{3}{c|}{$h / a=0.1$} & \multicolumn{3}{c|}{$h / a=0.2$} \\
\hline $\begin{array}{c}\text { Present } \\
\text { study }\end{array}$ & $\begin{array}{c}\text { Ref. } \\
{[5]}\end{array}$ & $\begin{array}{c}\text { Exact } \\
{[14]}\end{array}$ & $\begin{array}{c}\text { Present } \\
\text { study }\end{array}$ & $\begin{array}{c}\text { Ref. } \\
{[5]}\end{array}$ & $\begin{array}{c}\text { Exact } \\
{[14]}\end{array}$ & $\begin{array}{c}\text { Present } \\
\text { study }\end{array}$ & $\begin{array}{c}\text { Ref. } \\
{[5]}\end{array}$ & $\begin{array}{c}\text { Exact } \\
{[14]}\end{array}$ \\
\hline 0.0158 & 0.0147 & 0.0153 & 0.0621 & 0.0592 & 0.0596 & 0.2306 & 0.2188 & 0.2192 \\
\hline
\end{tabular}

Table 1. Dimensionless fundamental frequency $\left(\bar{\omega}=\omega h \sqrt{\frac{\rho_{m}}{E_{m}}}\right)$ of a simply supported square $\left(\mathrm{Al} / \mathrm{Zro}_{2}\right)$ FG plate $(p=1)$. 
case, there is insignificant difference between the result predicted by SSDT and TSDT; SSDT slightly over predicts frequencies. It can be seen that there are good agreements between our results and other results.

\begin{tabular}{|c|c|c|c|c|c|c|c|c|}
\hline \multicolumn{3}{|c|}{$p=2$} & \multicolumn{3}{c|}{$p=3$} & \multicolumn{3}{c|}{$p=5$} \\
\hline $\begin{array}{c}\text { Present } \\
\text { study }\end{array}$ & $\begin{array}{c}\text { Ref. } \\
{[5]}\end{array}$ & $\begin{array}{c}\text { Exact } \\
{[14]}\end{array}$ & $\begin{array}{c}\text { Present } \\
\text { Study }\end{array}$ & $\begin{array}{c}\text { Ref. } \\
{[5]}\end{array}$ & $\begin{array}{c}\text { Exact } \\
{[14]}\end{array}$ & $\begin{array}{c}\text { Present } \\
\text { Study }\end{array}$ & $\begin{array}{c}\text { Ref. } \\
{[5]}\end{array}$ & $\begin{array}{c}\text { Exact } \\
{[14]}\end{array}$ \\
\hline 0.2292 & 0.2188 & 0.2197 & 0.2306 & 0.2202 & 0.2211 & 0.2324 & 0.2215 & 0.2225 \\
\hline
\end{tabular}

Table 2. Dimensionless fundamental frequency $\left(\bar{\omega}=\omega h \sqrt{\frac{\rho_{m}}{E_{m}}}\right)$ of a simply supported square $\left(\mathrm{Al} / \mathrm{Zro}_{2}\right)$ FG Plate, thickness-to-side is: $h / a=0.2$.

\begin{tabular}{|c|c|c|c|}
\hline Material property & $E(\mathrm{Gpa})$ & $\rho\left(\mathrm{Kg} / \mathrm{m}^{3}\right)$ & $v$ \\
\hline SUS 304, Metal & 201.04 & 8166 & 0.33 \\
\hline Aluminum, Metal & 68.9 & 2700 & 0.33 \\
\hline Zirconia, Ceramic & 211.0 & 4500 & 0.33 \\
\hline $\mathrm{Si}_{3} \mathrm{~N}_{4}$, Ceramic & 348.43 & 2370 & 0.24 \\
\hline
\end{tabular}

Table 3. Properties of materials used in the numerical example.

\subsection{Numerical example}

For numerical illustration of the free vibration of a quadrangle FG plate with Zirconia and silicon nitride as the upper-surface ceramic and aluminum and SUS 304 as the lower-surface metal are considered the same as [10]:

\subsubsection{Results and discussion for the first ten modes in quadrangular FG plates}

In the following Tables, free vibrations are presented in dimensionless form for square and rectangular FG plates.

Tables 4 and 5 show the dimensionless frequency in square $(a=b)$ SUS 304/Si3N4, FG plates. It can be noted that for the same values of grading index $P$, the natural frequency increases with increasing mode. The effect of grading index can be shown by comparing the frequency value for the fixed value of mode and changing the values of grading index $p$. It can be seen that, the frequency decreases with the increase of the grading index due to the stiffness decreases from pure ceramic to pure metal.

Tables 6 and 7 show the dimensionless frequency in rectangular $(b=2 a)$ SUS 304/Si3N4, FG plates. The effect of grading index can be shown by comparing the frequency for the same value of mode and considering different values of grading index $p$ as shown in Table 5. It is clearly visible that the frequency decreases with the increasing grading index, caused by the stiffness decreasing with increasing grading index. For the same value of $p$, it can be said that the natural frequency increases with increasing mode. By comparing Tables 6, 7 and 4, 5 it can be observed that for the same values of grading index and mode, the fundamental frequency in square FG plates are greater than those in rectangular FG plates and by 


\begin{tabular}{|c|c|c|c|c|c|c|c|c|c|}
\hline$m \times n$ & mode & $p=0$ & $p=0.5$ & $p=1$ & $p=2$ & $p=4$ & $p=6$ & $p=8$ & $p=10$ \\
\hline $1 \times 1$ & 1 & 5.76 & 3.904 & 3.393 & 3.027 & 2.795 & 2.697 & 2.638 & 2.597 \\
\hline $1 \times 2$ & 2 & 13.846 & 9.366 & 8.139 & 7.259 & 6.700 & 6.464 & 6.323 & 6.227 \\
\hline $2 \times 1$ & 3 & 13.846 & 9.366 & 8.139 & 7.259 & 6.700 & 6.464 & 6.323 & 6.227 \\
\hline $2 \times 2$ & 4 & 21.353 & 14.441 & 12.547 & 11.187 & 10.321 & 9.957 & 9.741 & 9.593 \\
\hline $2 \times 3$ & 5 & 32.859 & 22.220 & 19.305 & 17.203 & 15.863 & 15.300 & 14.967 & 14.741 \\
\hline $3 \times 2$ & 6 & 32.859 & 22.220 & 19.305 & 17.203 & 15.863 & 15.300 & 14.967 & 14.741 \\
\hline $3 \times 3$ & 7 & 43.369 & 29.323 & 25.472 & 22.689 & 20.911 & 20.167 & 19.729 & 19.431 \\
\hline $3 \times 4$ & 8 & 56.798 & 38.405 & 33.362 & 29.703 & 27.356 & 26.377 & 25.801 & 25.412 \\
\hline $4 \times 3$ & 9 & 56.798 & 38.405 & 33.362 & 29.703 & 27.356 & 26.377 & 25.801 & 25.412 \\
\hline $4 \times 4$ & 10 & 69.054 & 46.690 & 40.555 & 36.091 & 33.221 & 32.026 & 31.327 & 30.856 \\
\hline
\end{tabular}

Table 4. Variation of the frequency parameter $\left(\varpi=\omega a^{2} / h \sqrt{\rho_{c} / E_{c}}\right)$ with the grading index ( $p$ ) for square. SUS $304 / S i_{3} N_{4}$ FG square plates $(a / h=10, a=b)$.

\begin{tabular}{|c|c|c|c|c|c|c|c|c|c|}
\hline$m \times n$ & mode & $p=0$ & $p=0.5$ & $p=1$ & $p=2$ & $p=4$ & $p=6$ & $p=8$ & $. p=10$. \\
\hline $1 \times 1$ & 1 & 5.338 & 3.610 & 3.137 & 2.796 & 2.580 & 2.489 & 2.435 & 2.398 \\
\hline $1 \times 2$ & 2 & 11.836 & 8.003 & 6.953 & 6.193 & 5.706 & 5.502 & 5.382 & 5.301 \\
\hline $2 \times 1$ & 3 & 11.836 & 8.003 & 6.953 & 6.193 & 5.706 & 5.502 & 5.382 & 5.301 \\
\hline $2 \times 2$ & 4 & 17.263 & 11.672 & 10.138 & 9.022 & 8.305 & 8.006 & 7.831 & 7.714 \\
\hline $2 \times 3$ & 5 & 24.881 & 16.828 & 14.621 & 13.002 & 11.950 & 11.513 & 11.258 & 11.089 \\
\hline $3 \times 2$ & 6 & 24.881 & 16.828 & 14.621 & 13.002 & 11.950 & 11.513 & 11.258 & 11.089 \\
\hline $3 \times 3$ & 7 & 31.354 & 21.209 & 18.426 & 16.375 & 15.0343 & 14.477 & 14.156 & 13.943 \\
\hline $3 \times 4$ & 8 & 39.180 & 26.508 & 23.041 & 20.471 & 18.770 & 18.062 & 17.656 & 17.388 \\
\hline $4 \times 3$ & 9 & 39.180 & 26.508 & 23.041 & 20.471 & 18.770 & 18.062 & 17.656 & 17.388 \\
\hline $4 \times 4$ & 10 & 46.020 & 31.141 & 27.067 & 24.036 & 22.020 & 21.181 & 20.702 & 20.387 \\
\hline
\end{tabular}

Table 5. Variation of the frequency parameter $\left(\varpi=\omega a^{2} / h \sqrt{\rho_{c} / E_{c}}\right)$ with the grading index $(p)$ for SUS $304 / S i_{3} N_{4}$ FG square plates $(a / h=5, a=b)$.

\begin{tabular}{|c|c|c|c|c|c|c|c|c|c|}
\hline$m \times n$ & mode & $p=0$ & $p=0.5$ & $p=1$ & $\cdot p=2$. & $p=4$ & $p=6$ & $p=8$ & $p=10$ \\
\hline $1 \times 1$ & 1 & 3.461 & 2.341 & 2.034 & 1.814 & 1.674 & 1.616 & 1.580 & 1.556 \\
\hline $1 \times 2$ & 2 & 5.338 & 3.610 & 3.137 & 2.796 & 2.580 & 2.489 & 2.435 & 2.39 \\
\hline $2 \times 1$ & 3 & 10.334 & 6.984 & 6.065 & 5.402 & 4.980 & 4.804 & 4.700 & \\
\hline $2 \times 2$ & 4 & 11.836 & 8.00 & 6.948 & 6.188 & 5.702 & 5.499 & 5.380 & 5.300 \\
\hline $2 \times 3$ & 5 & 14.199 & 9.599 & 8.337 & 7.422 & 6.836 & 6.592 & 6.449 & 6.552 \\
\hline $3 \times 2$ & 6 & 20.484 & 13.845 & 12.020 & 10.689 & 9.835 & 9.482 & 9.276 & 9.139 \\
\hline $3 \times 3$ & 7 & 22.373 & 15.125 & 13.133 & 11.678 & 10.740 & 10.352 & 10.126 & 9.976 \\
\hline $3 \times 4$ & 8 & 24.881 & 16.824 & 14.611 & 12.989 & 11.940 & 11.505 & 11.254 & 11.085 \\
\hline $4 \times 3$ & 9 & 31.656 & 21.409 & 18.585 & 16.506 & 15.157 & 14.602 & 14.282 & 14.071 \\
\hline $4 \times 4$ & 10 & 33.715 & 22.805 & 19.802 & 17.587 & 16.142 & 15.547 & 15.205 & 14.979 \\
\hline
\end{tabular}

Table 6. Variation of the frequency parameter $\left(\varpi=\omega a^{2} / h \sqrt{\rho_{c} / E_{c}}\right)$ with the grading index ( $p$ ) for SUS $304 / S i_{3} N_{4}$ FG rectangular plate $(a / h=5, a=0.5 \times b)$. 


\begin{tabular}{|c|c|c|c|c|c|c|c|c|c|}
\hline$m \times n$ & mode & $p=0$ & $p=0.5$ & $p=1$ & $p=2$ & $p=4$ & $p=6$ & $p=8$ & $p=10$ \\
\hline $1 \times 1$ & 1 & 3.645 & 2.467 & 2.144 & 1.913 & 1.766 & 1.704 & 1.667 & 1.642 \\
\hline $1 \times 2$ & 2 & 5.769 & 3.904 & 3.393 & 3.027 & 2.795 & 2.697 & 2.638 & 2.597 \\
\hline $2 \times 1$ & 3 & 11.885 & 8.039 & 6.986 & 6.231 & 5.752 & 5.549 & 5.429 & 5.346 \\
\hline $2 \times 2$ & 4 & 13.846 & 9.365 & 8.138 & 7.258 & 6.699 & 6.463 & 6.323 & 6.227 \\
\hline $2 \times 3$ & 5 & 17.037 & 11.523 & 10.012 & 8.928 & 8.239 & 7.949 & 7.776 & 7.658 \\
\hline $3 \times 2$ & 6 & 26.092 & 17.640 & 15.325 & 13.659 & 12.600 & 12.156 & 11.893 & 11.713 \\
\hline $3 \times 3$ & 7 & 28.958 & 19.578 & 17.008 & 15.158 & 13.981 & 13.487 & 13.195 & 12.995 \\
\hline $3 \times 4$ & 8 & 32.859 & 22.215 & 19.299 & 17.197 & 15.858 & 15.297 & 14.965 & 14.739 \\
\hline $4 \times 3$ & 9 & 43.873 & 29.653 & 25.754 & 22.937 & 21.142 & 20.393 & 19.951 & 19.652 \\
\hline $4 \times 4$ & 10 & 47.344 & 32.002 & 27.794 & 24.715 & 22.809 & 21.999 & 21.522 & 21.199 \\
\hline
\end{tabular}

Table 7. Variation of the frequency parameter $\left(\varpi=\omega a^{2} / h \sqrt{\rho_{c} / E_{c}}\right)$ with the grading index $(p)$ for SUS $304 / S_{3} N_{4}$ FG rectangular plate $(a / h=10, a=0.5 \times b)$.

increasing the side-to-thickness ratio, the frequency also increases. It is evident that the grading index and side-to-thickness ratio effects in frequency are more significant than the other conditions.

\subsubsection{Results and discussion for the natural frequency in quadrangular FG (SUS 304/Si3N4) plates}

Figures (3) and (4) illustrate the dimensionless frequency versus grading index $(p)$, for different values of side-to-thickness ratio $(a / h)$ and side-to-side ratio $(b / a)$, respectively. In Figure 3 , the effect of grading index $(p)$ and side-to-thickness ratio $(a / h)$ on dimensionless fundamental frequency of FG (SUS 304/Si3N4) plate is shown. It can be seen that the frequency decreases with increasing grading index, due to degradation of stiffness by the metallic inclusion. It can be observed that the natural frequency is maximum for fullceramic $(p=0.0)$ and this value increases with the increase of the side-to-thickness ratio, since the stiffness of thin plates is more effectively than the thick plates. It is seen that for the values $(p)$, for $0<p<2$ the slope is greater than other parts $(p>2)$. It can be said that for side-to-thickness ratios greater than twenty $(a / h>20)$, the frequencies will be similar for different values of grading index. It can be noted that the difference between frequencies in $a / h=5$ and $a / h=10$ are greater than differences of frequency between $a / h=10$ and other curves for the same values of grading index $p$. And also it can be concluded that for $a / h>20$, the difference between the frequencies is small for the same value of grading index.

The effect of grading index $(p)$ and side-to-side ratio $(b / a)$ on dimensionless fundamental frequency of FG (SUS 304/Si3N4) plate can be seen in figure 4 . It can be noted that the frequency increases with the increase of the $b / a$ since rectangular plates can be treated as a one-dimensional problem for example, beams or plate strips. It can be observed that the frequency is almost constant for different values of grading index. 


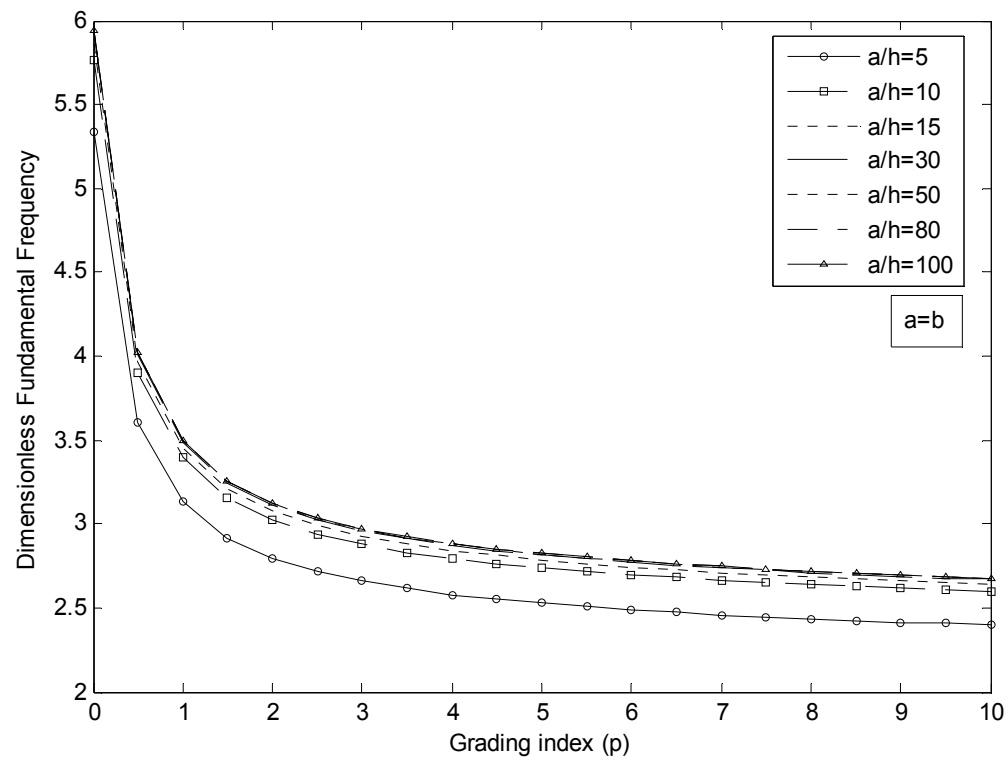

Fig. 3. Dimensionless frequency $\left(\varpi=\omega a^{2} / h \sqrt{\rho_{c} / E_{c}}\right)$ versus grading index $(p)$ for different values of side-to-thickness ratio $(a / h)$ in square $(b=a)$ FG (SUS $\left.304 / S i_{3} N_{4}\right)$ plates.

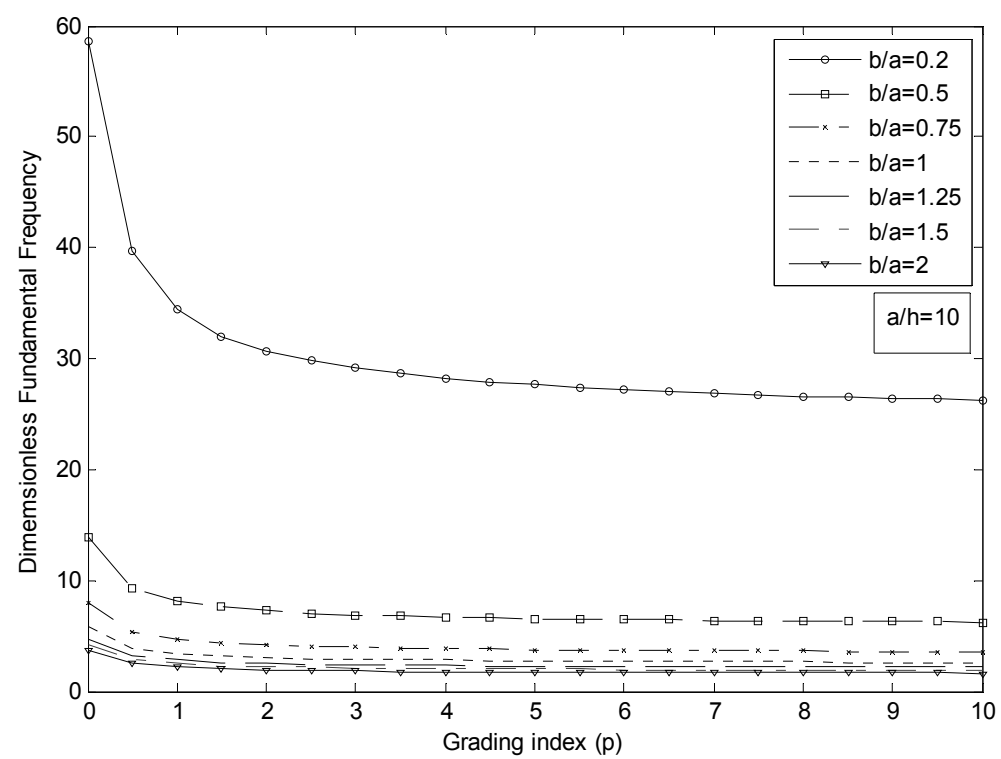

Fig. 4. Dimensionless frequency $\left(\varpi=\omega a^{2} / h \sqrt{\rho_{c} / E_{c}}\right)$ versus grading index $(p)$ for different values of side-to-side ratio $(b / a)$ FG ( SUS $\left.304 / S i_{3} N_{4}\right)$ plates when $a / h=10.0$ 
Figures (5) and (6) show variation of dimensionless fundamental frequency of FG (SUS $304 / \mathrm{Si} 3 \mathrm{~N} 4)$ plate with side-to-thickness ratio $(a / h)$, for different values of grading index $(p)$ and side-to side ratio $(b / a)$, respectively.

It is seen from figure 5 , the fundamental frequency increases with the increase of the value of side-to-thickness ratio $(a / h)$. It is shown that the frequency decreases with the increase of the values of side-to-side $(b / a)$. It can be noted that the slope of frequency versus sideto-thickness ratio $(a / h)$ for part $5<a / h<10$ is greater than those in another part $(a / h>10)$.

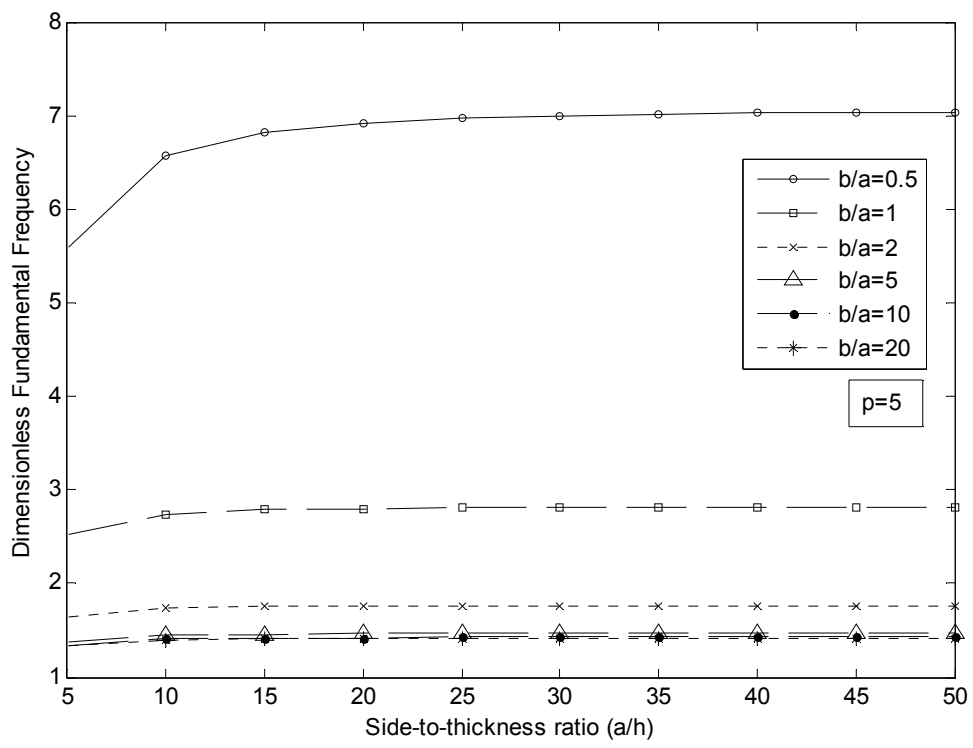

Fig. 5. Dimensionless frequency $\left(\varpi=\omega a^{2} / h \sqrt{\rho_{c} / E_{c}}\right)$ versus side-to-thickness ratio $(a / h)$ for different values of side-to-side ratio $(b / a)$ FG (SUS $\left.304 / S i_{3} N_{4}\right)$ plates when $p=5$. 


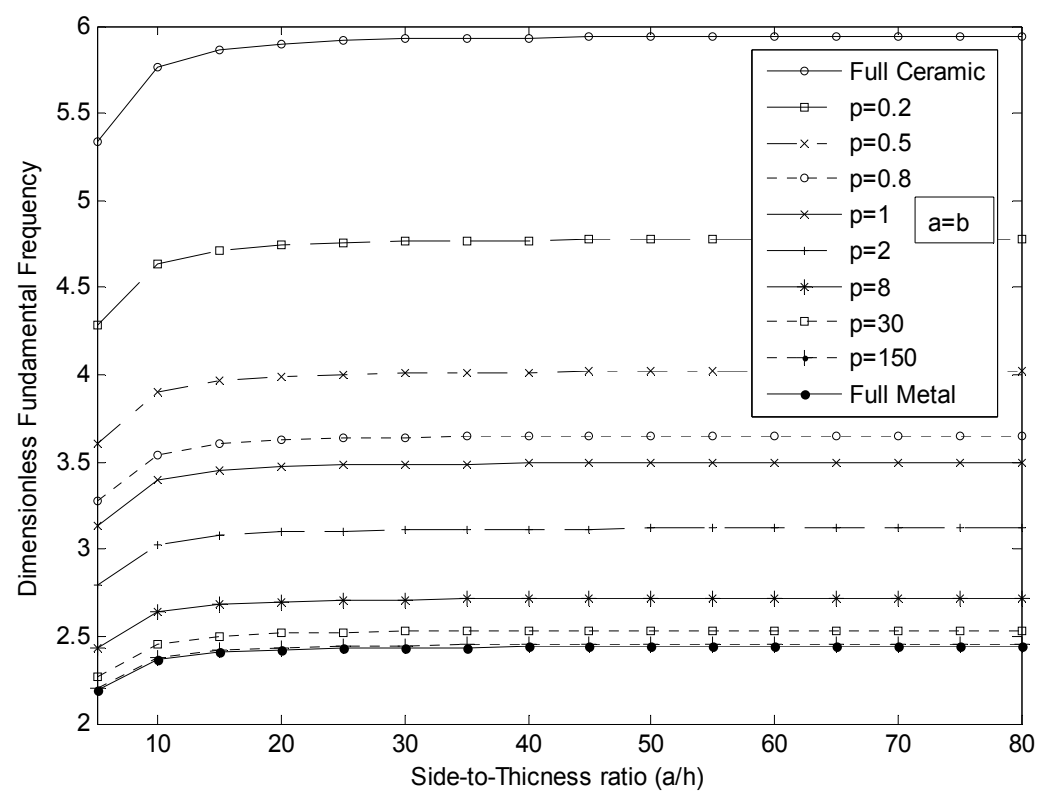

Fig. 6. Dimensionless frequency $\left(\varpi=\omega a^{2} / h \sqrt{\rho_{c} / E_{c}}\right)$ versus side-to-thickness ratio $(a / h)$ for different values of grading index $(p)$ in square $(b=a) \mathrm{FG}$ (SUS $\left.304 / S i_{3} N_{4}\right)$ plates.

The variation of frequency with side-to-thickness ratio $(a / h)$ for different values of grading index $(p)$ is presented in Figure 6. As expected, by increasing the value of grading index $(p)$ the values of frequency decrease due to the decrease in stiffness. Similarly, in figure (5) while the $5<a / h<10$, the slope is greater than another ratios. It can be noted that for the values of grading index $p>30$, the results for frequency are similar.

Figures 7 and 8 present the variation of dimensionless frequency of FG (SUS 304/Si3N4) plate versus side-to-side ratio $(b / a)$ for different values of grading index $(p)$ and side-tothickness ratio $(a / h)$, respectively. 


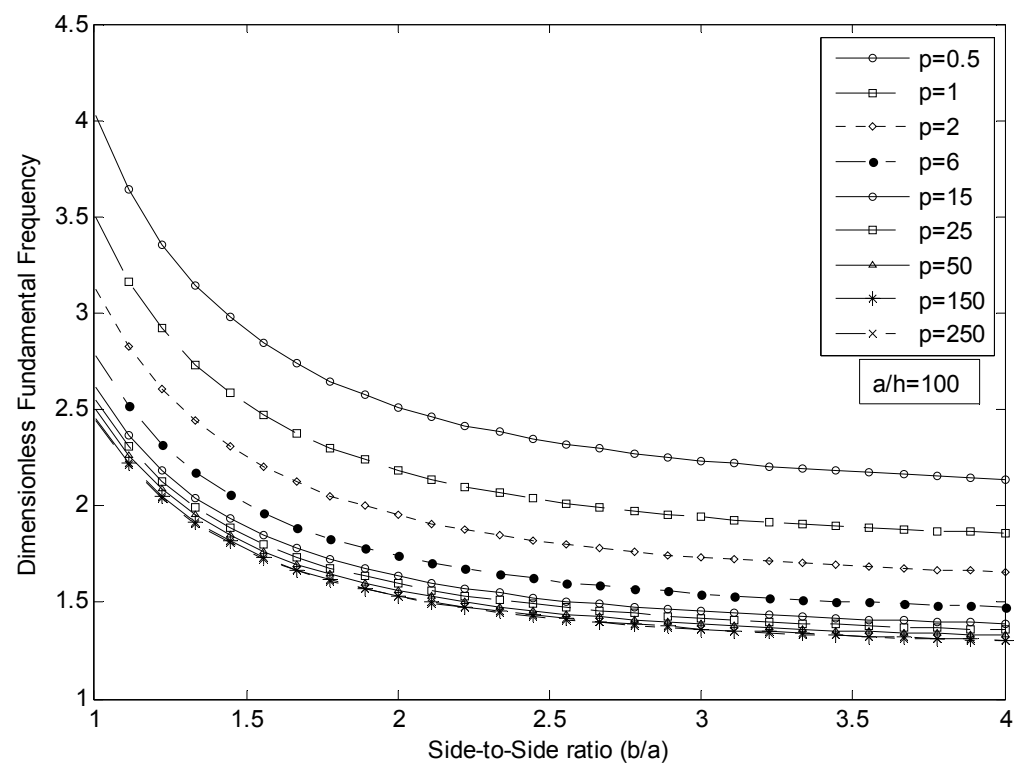

Fig. 7. Dimensionless frequency $\left(~ \varpi=\omega a^{2} / h \sqrt{\rho_{c} / E_{c}}\right)$ versus side-to-side ratio $(b=a)$ for different values of grading index $(p) \mathrm{FG}\left(\right.$ SUS $\left.304 / S i_{3} N_{4}\right)$ plates when $a / h=100$.

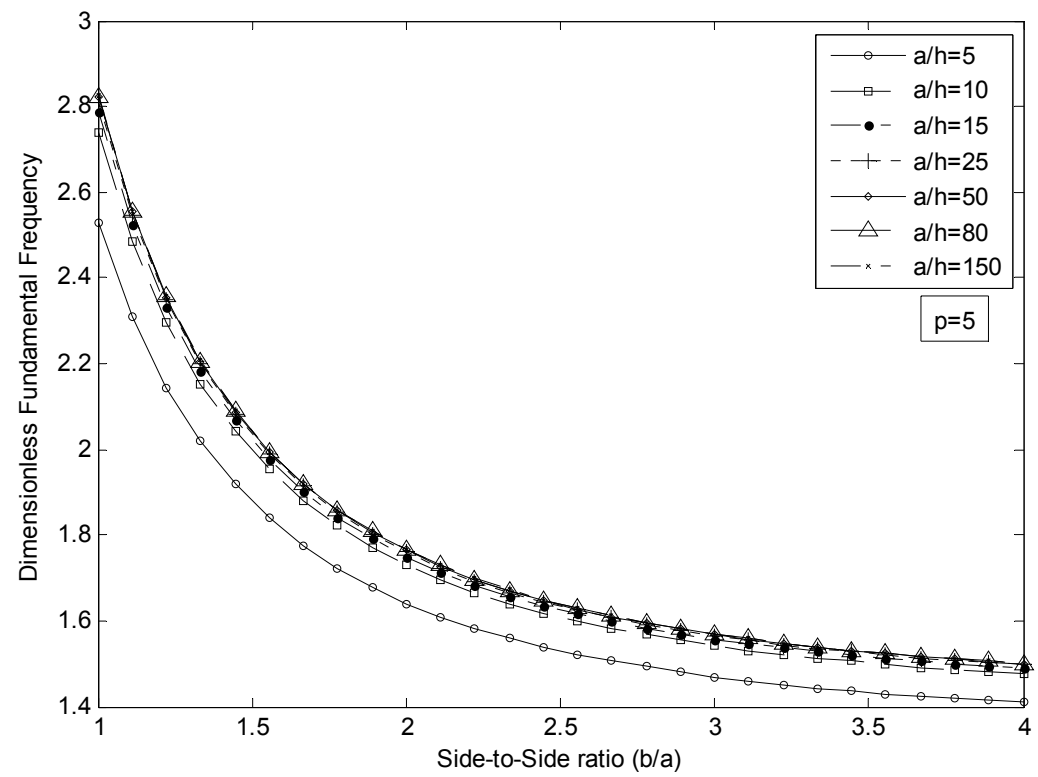

Fig. 8. Dimensionless frequency $\left(\varpi=\omega a^{2} / h \sqrt{\rho_{c} / E_{c}}\right)$ versus side-to-side ratio $(b / a)$ for different values of side-to-thickness ratio $(a / h)$ FG ( SUS $\left.304 / S i_{3} N_{4}\right)$ plates when $p=5$. 
In figure 7, it is shown that the frequency decreases with the increase of the value of side-toside ratio $(b / a)$ for all values of grading index $(p)$. It is seen that the frequencies for FG quadrangular plates are between that of a full-ceramic plate and full-metal plate. As expected the frequencies in a full-ceramic plate are greater than those in a full-metal plate.

The results for dimensionless frequency versus side-to-side ratio $(b / a)$ for different values of side-to-thickness ratio $(a / h)$ in FG plate while grading index $p=5$ are shown in figure 8 . It is seen that by increasing the value of $b / a$, the frequency decreases for all values of $a / h$. It can be noted for $a / h>10$ the results are similar.

\section{Conclusions}

In this chapter, free vibration of FG quadrangular plates were investigated thoroughly by adopting Second order Shear Deformation Theory (SSDT). It was assumed that the elastic properties of a FG quadrangular plate varied along its thickness according to a power law distribution. Zirconia and Si3N4 were considered as a ceramic in the upper surface while aluminum and SUS304 were considered as metals for the lower surface. The complete equations of motion were presented using Hamilton's principle. The equations were solved by using Navier's Method for simply supported FG plates.

Some general observations of this study can be deduced here:

- The decreasing slope of the fundamental frequency for $0<p<2$, is greater than another part $(p>2)$ for all values of side-to-thickness ratio $(a / h)$ in square FG plate.

- It was found that the fundamental frequency of the FG plate increases with the increase of the value of side-to-side ratio $(b / a)$.

- For FG plates, the slope of increasing frequency versus side-to-thickness $(a / h)$ when $5<a / h<10$ is greater than another part $(a / h>10)$ for any value of grading index and side-to-side ratio.

- The fundamental frequency versus side-to-side ratio $(b / a)$ for FG quadrangular plates are between those of a full-ceramic plate and full-metal plate when $a / h=10$.

From the numerical results presented here, it can be proposed that the gradations of the constitutive components are the significant parameter in the frequency of quadrangular FG plates.

\section{Acknowledgement}

The authors would like to thank Universiti Putra Malaysia for providing the research grant (FRGS 07-10-07-398SFR 5523398) for this research work.

\section{References}

[1] Reddy JN. Analysis of functionally graded plates. Int. J. Numer Meth Eng 2000;47:663-684.

[2] Suresh S, Mortensen A. Fundamentals of functionally graded materials. London: IOM Communications Limited, 1998.

[3] Praveen GN, Reddy JN. Nonlinear transient thermoelastic analysis of functionally graded ceramic-metal plates. Int. J. Solids Struct 1998;35(33):4457-4476. 
[4] Ng TY, Lam KY, Liew KM. Effects of FGM materials on the parametric resonance of plate structure. Comput. Meth Appl. Mech. Eng 2000;190:953-962.

[5] Ferreira AJM, Batra RC, Roque CMC, Qian LF, Jorge RMN. Natural frequencies of functionally graded plates by a meshless method. Comp Struct 2006;75:593-600.

[6] Woo J, Meguid SA, Ong LS. Nonlinear free vibration behavior of functionally graded plates. J. Sound Vibr 2006;289:595-611.

[7] Zhao X, Lee YY, Liew KM. Free vibration analysis of functionally graded plates using the element-free kp-Ritz method. J. Sound Vibr 2008.

[8] Batra RC, Jin J. Natural frequencies of a functionally graded anisotropic rectangular plate. J. Sound Vibr 2005;282:509-516.

[9] Batra RC, Aimmanee S. Vibrations of thick isotropic plates with higher order shear and normal deformable plate theories. Comput Struct 2005;83:934-955.

[10] Bayat M, Saleem M, Sahari BB, Hamouda AMS, Mahdi E. Thermo elastic analysis of a functionally graded rotating disk with small and large deflections. Thin-Walled Struct 2007;45:677-691.

[11] Bayat M, Sahari BB, Saleem M, Ali A, Wong SV. Thermo elastic solution of a functionally graded variable thickness rotating disk with bending based on the first-order shear deformation theory. Thin-Walled Struct 2008.

[12] Heidary F, M. Reza Eslami MR. Piezo-control of forced vibrations of a thermoelastic composite plate. Comp Struct 2006;74(1):99-105

[13] Cheng ZQ, Batra RC. 2000, exact correspondence between eigenvalue of membranes and functionally graded simply supported polygonal plates. J. Sound Vibr 2000;229(4):879-895.

[14] Vel SS, Batra RC. Three-dimensional exact solution for the vibration of functionally graded rectangular plates. J. Sound Vibr 2004;272: 703-30.

[15] Huang XL, Shen $H$. Nonlinear vibration and dynamic response of functionally graded plates in thermal environments. Int J. Solids Struct 2004;41:2403-2427.

[16] Ferreira AJM., Batra RC, Roque CMC, Qian LF, Martins PALS. Static analysis of functionally graded plates using third-order shear deformation theory and meshless method. Comp Struct 2005;69:449-457.

[17] Khdeir AA, Reddy JN. Free vibrations of laminated composite plates using secondorder shear deformation theory. Comp Struct 1999;71:617-626.

[18] Bahtui A, Eslami MR. Coupled thermoelasticity of functionally graded cylindrical shells. Mech Res Commun 2007; 34(1):1-18.

[19] Reddy JN. Theory and Analysis of Elastic Plates and Shells. New York: CRC Press; 2007.

[20] Stoffel, M. (2005). Experimental validation of simulated plate deformations caused by shock waves, Math. Mech., 85(9):643 - 659.

[21] Saidi Ali Reza, Sahraee Shahab, (2006). Axisymmetric solutions of functionally graded circular and annular plates using second-order shear deformation plate theory, ESDA2006-95699, 8th Biennial ASME Conference on Engineering Systems Design and Analysis, Torino, Italy. 
[22] Librescu,L., Khdeir, A.A., Reddy, J.N. (1987). Comprehensive Analysis of the State of Stressof Elastic Anisotropie Flat Plates Using Refined Theories, Acta Mechanica, 70:57-81.

[23] Librescu, L., Schmidt, R. (1988).Refined Theories of Elastic Anisotropic Shell Accounting for Small Strain and Moderate Rotations, Int. J. Non-Linear Mechanics, 23(3):217-229. 


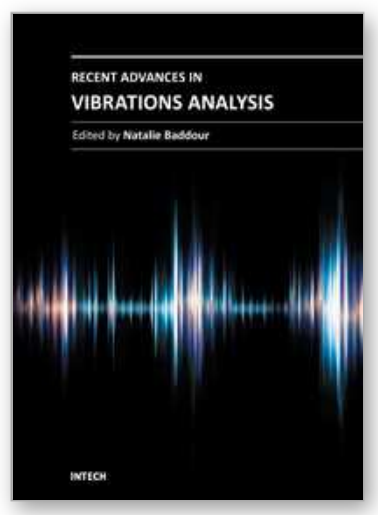

\author{
Recent Advances in Vibrations Analysis \\ Edited by Dr. Natalie Baddour
}

ISBN 978-953-307-696-6

Hard cover, 236 pages

Publisher InTech

Published online 09, September, 2011

Published in print edition September, 2011

This book covers recent advances in modern vibrations analysis, from analytical methods to applications of vibrations analysis to condition monitoring. Covered topics include stochastic finite element approaches, wave theories for distributed parameter systems, second other shear deformation theory and applications of phase space to the identifications of nonlinearities and transients. Chapters on novel condition monitoring approaches for reducers, transformers and low earth orbit satellites are included. Additionally, the book includes chapters on modelling and analysis of various complex mechanical systems such as eccentric building systems and the structural modelling of large container ships.

\title{
How to reference
}

In order to correctly reference this scholarly work, feel free to copy and paste the following:

A. Shahrjerdi and F. Mustapha (2011). Second Order Shear Deformation Theory (SSDT) for Free Vibration Analysis on a Functionally Graded Quadrangle Plate, Recent Advances in Vibrations Analysis, Dr. Natalie Baddour (Ed.), ISBN: 978-953-307-696-6, InTech, Available from: http://www.intechopen.com/books/recentadvances-in-vibrations-analysis/second-order-shear-deformation-theory-ssdt-for-free-vibration-analysis-on-afunctionally-graded-quad

\section{INTECH}

open science | open minds

\section{InTech Europe}

University Campus STeP Ri

Slavka Krautzeka 83/A

51000 Rijeka, Croatia

Phone: +385 (51) 770447

Fax: +385 (51) 686166

www.intechopen.com

\section{InTech China}

Unit 405, Office Block, Hotel Equatorial Shanghai

No.65, Yan An Road (West), Shanghai, 200040, China 中国上海市延安西路65号上海国际贵都大饭店办公楼 405 单元

Phone: +86-21-62489820

Fax: $+86-21-62489821$ 
(C) 2011 The Author(s). Licensee IntechOpen. This chapter is distributed under the terms of the Creative Commons Attribution-NonCommercialShareAlike-3.0 License, which permits use, distribution and reproduction for non-commercial purposes, provided the original is properly cited and derivative works building on this content are distributed under the same license. 